\title{
Risk Factors for Developmental Dysplasia of the Hip in the Newborn Data from the Swedish Medical Birth Register
}

\section{Bengt Kallen*}

Tornblad Institute, Biskopsgatan 7, SE-223 63 Lund, Sweden

\begin{abstract}
Background: Developmental dysplasia of the hip (DDH) is a common anomaly in the neonate which, if not treated, can result in chronic hip problems. In the previous literature some risk factors are known: female sex, first parity, breech presentation and family history.

Material and methods: The Swedish Medical Birth Register was used to identify infants with neonatal DDH for the period 1973-2011 and data from 1998-2011 were used in order to identify and quantify risk factors. Analysis was performed with Mantel-Haenszel methodology with estimates of odds ratios and approximate $95 \%$ confidence intervals were estimated with Miettinen's method.

Results: Among all infants born ( $n=3,977,681), 34,530$ had DDH. The rate of DDH diagnoses declined markedly during the 1980s but was relatively constant after 1998. A marked geographical variation was found. The previously identified risk factors were verified: female sex, breech presentation, family history, first parity. An increasing risk with maternal age (adjusted for parity) was seen and a reduced risk at maternal smoking. There is a strong linear regression between gestational duration or birth weight and presence of DDH while only reduced and not excess intrauterine growth markedly affected DDH rate. Caesarean section did not change the DDH risk at vertex presentation but reduced it at breech presentation. Maternal use of anticonvulsants increased the risk for DDH but a decreased risk was seen after maternal use of insulin or antidepressants.
\end{abstract}

Conclusions: The study has demonstrated previously little known risk factors for DDH.

Keywords: Developmental dysplasia of hip; Risk factors; Maternal smoking; Anticonvulsant use; Pregnancy duration; Birth weight

Abbreviations: BMI: Body Mass Index; CI: Confidence Interval; DDH: Developmental Dysplasia of Hip; OR: Odds Ratio; RR: Risk Ratio; SNRI/NRI: Serotonin/Noradrenalin Reuptake Inhibitor; SSRI: Selective Serotonin Reuptake Inhibitor

\section{Introduction}

Developmental dysplasia of the hip (DDH) contains a spectrum of conditions: abnormal acetabular shape, partial or incomplete displacement of the femoral head from the acetabulum, and unstable hip [1]. It is a relatively common condition but, if not identified and treated in the neonate, it can in some instances lead to long-term hip problems. In Sweden all neonates have been screened for DDH by qualified pediatricians and when the findings have been abnormal, early correction has been applied. Some well known risk factors for DDH exist and have been summarized in two recent reviews $[1,2]$. These are infant sex (female excess), breach presentation, family history, and in some studies being first born. Methods of delivery (vaginal vs. caesarean section) gave no statistically significant effect. Low birth weight appeared as a "protective" factor but there was no significant effect of a high birth weight [2]. One small study found an association between hyperthyroidism and DDH [3].

The present study uses the Swedish Medical Birth Register and studies the effect of various factors on the occurrence of a diagnosis of DDH in the newborn [4].

\section{Material and Methods}

The study was made on deliveries in Sweden 1973-2011 but analyses of risk factors were restricted to the period 1998-2011.

\section{The following risk factors were studied}

- Geography defined as the county where the mother lived at delivery (and in most instances the birth took place).

- Year of delivery.

- Maternal age in 5-year classes $(<20,20-24$ etc. $)$

- Parity $(1 \geq 4)$ where parity $1=$ first child born by the mother.

- Smoking based on midwife interview towards the end of the first trimester (unknown, none, $<10$ cigarettes/day, $\geq 10$ cigarettes per day).

- Body mass index calculated from prepregnancy weight and length, obtained at the midwife interview (unknown, <18.5, 18.5-24.9, 25-29.9, 30-34.9, $\geq 35$ ).

- Unwanted childlessness (none, 1, 2, 3, 4, $\geq 5$ years).

- Maternal use of drugs during early pregnancy, from the midwife interviews.

- Presentation (vertex, breech, other).

- Method of delivery (vaginal non-instrumental, vaginal instrumental, caesarean section).

- Infant sex.

*Corresponding author: Bengt Kallen, Tornblad Institute, Biskopsgatan 7, SE22363 Lund, Sweden, Tel: 46-46-222 7536; Fax: +46-46-222 4226; E-mail: Bengt.Kallen@med.lu.se

Received September 18, 2013; Accepted September 20, 2013; Published September 25, 2013

Citation: Kallen B (2014) Risk Factors for Developmental Dysplasia of the Hip in the Newborn Data from the Swedish Medical Birth Register. Clinics Mother Child Health 11: 153. doi: 10.4172/2090-7214.1000153

Copyright: (c) 2014 Kallen B. This is an open-access article distributed under the terms of the Creative Commons Attribution License, which permits unrestricted use, distribution, and reproduction in any medium, provided the original author and source are credited. 
- Pregnancy duration in weeks among singletons, usually determined by second trimester sonography.

- Birth weight among singletons.

- Intrauterine growth disturbances among singletons based on sex and parity specific growth graphs from the Medical Birth Register [5].

The Medical Birth Register contains information on practically all women who give birth in Sweden (1-2\% is missing). Individuals in the register are identified with their ID numbers. All persons living in Sweden have such numbers which are widely used in society and in all health documents. The Id number contains the date of birth of the individual. The presence of DDH among the infants was identified from diagnoses given by qualified pediatricians.

Using the Id. number of the mothers of infants with DDH, such women were identified, born in 1973-1994. These women's own delivery records were identified in the register and the presence of DDH ascertained. The rate of DDH among infants born of these women was compared with the rate of DDH among other female infants, born 1973-1994. Adjustment was made for year of birth, maternal age, and parity. Smoking information was not available until in 1982.

Adjustment for co-variates was made with the Mantel-Haenszel methodology. Odds ratios (OR) were estimated and approximate 95\% confidence intervals (95\%CI) were calculated using Miettinen's method. When the expected number of outcome was $<10$, risk ratios (RR) were instead calculated as observed/expected number and its $95 \%$ CI was estimated from exact Poisson distributions. Graph fitting was made with the program Analyse-it, Analyse-it Software, Ltd, Leeds UK.

In order to look for yearly and geographical variation, crude analyses were made.

\section{Results}

\section{Annual and geographical variations in rates of $\mathrm{DDH}$}

A total of 34,530 infants with DDH were identified for the whole study period (1973-2011) among 3,977,681 infants (8.7 per 1000). During 1998-2011 (the period when risk factor analysis was made) 8,092 infants had DDH among 1,392,126 infants born (6.3 per 1000).

Figure 1 shows that the rate of the diagnosis DDH was relatively constant up to the middle of the 1980s when a decline began. The graph planned out ten years later. In the adjusted analysis for the period 19982011 two-year periods were used (Table 1). Only minor differences

\begin{tabular}{|l|c|c|c|}
\hline Malformation group & Number (expected) & $\mathbf{R R}$ & $\mathbf{9 5 \%} \mathbf{~ C l}$ \\
\hline CNS malformations & $16(4.3)$ & 3.72 & $2.13-6.04^{\#}$ \\
\hline Ear/branchial malformations & $23(16.1)$ & 1.43 & $0.95-2.15$ \\
\hline Cardiovascular defects & $108(71.0)$ & 1.52 & $1.26-1.83$ \\
\hline Orofacial clefts & $20(11.4)$ & 1.75 & $1.14-2.70$ \\
\hline Alimentary tract atresia & $15(5.4)$ & 2.78 & $1.55-4.58^{\#}$ \\
\hline Hypospadias & $20(10.7)$ & 1.87 & $1.21-2.88$ \\
\hline Undescended testicle & $47(18.2)$ & 2.58 & $1.96-3.40$ \\
\hline Positional foot defect & $51(12.4)$ & 4.11 & $3.10-5.30$ \\
\hline Poly- or syndactyly & $22(12.8)$ & 1.72 & $1.14-2.60$ \\
\hline Nevus & $18(14.3)$ & 1.26 & $0.9-2.00$ \\
\hline
\end{tabular}

"Exact $95 \% \mathrm{Cl}$ from Poisson distributions.

Expected numbers from presence of the malformation in all infants and crude risk ratios (RR) as observed/expected numbers with $95 \%$ confidence intervals (95\% Cl).

Table 1: Concomitant congenital malformations among infants with $\mathrm{DDH}$. between years were seen for that period, but the last two years showed a significantly low rate.

Figure 2 compares the rates of DDH in the different counties in Sweden, 1998-2011. One group of counties had an average rate of 3.0 per 1000 , the majority 5.4 per 1000 , and one group 8.4 per 1000 . The very low rates in some counties are based on few cases. Adjustment will be made for these three groups.

When the rates were compared between different delivery hospitals, a weak trend $(\mathrm{p}=0.048)$ is seen with an increasing rate at increasing number of deliveries. Among the eight university hospitals the rates varied between 3.4 and 9.9 per 1000 .

\section{Characteristics of infants with DDH}

Among the infants with DDH 1998-2011, 27\% were males and 73\% females, sex ratio 0.37 (95\% CI $0.35-0.39)$ or 2.7 times higher rate in females than in males. The percentage of females showed no correlation with the rate of DDH but the percentage was higher around 1995 than earlier or later (Figure 3). There was no correlation between the county rate and the percentage of females $(\mathrm{p}=0.15)$.

Other congenital malformations were present in only 367 cases (4.5\%). Table 1 shows the number of infants with main groups of concomitant malformations compared with the rates among all infants registered in the Medical Birth Register, adjusted for infant sex. All types of tabulated malformations occurred in excess but it was especially marked for positional foot defects. For ear/branchial malformations and for nevus, the risks were not significant.

\section{Maternal and infant characteristics as risk factors}

Table 2 shows that the risk for a DDH diagnosis increased with maternal age and was higher at first parity than at higher parities. Maternal smoking resulted in a reduced risk while maternal BMI or subfertility (measured as years of unwanted childlessness) did not affect the risk significantly (Table 3 ). There were no clear-cut differences in these risk factors between male and female infants.

The risk for a DDH diagnosis according to pregnancy duration in singletons is shown in Figure 4 and according to birth weight in Figure 5. For both variables a relatively strong regression is seen. Figure 6 shows the relationship with intrauterine growth. The risk increases with increasing growth rate up to normal values but for higher growth rates no clear-cut further increase occurs. All these graphs are based

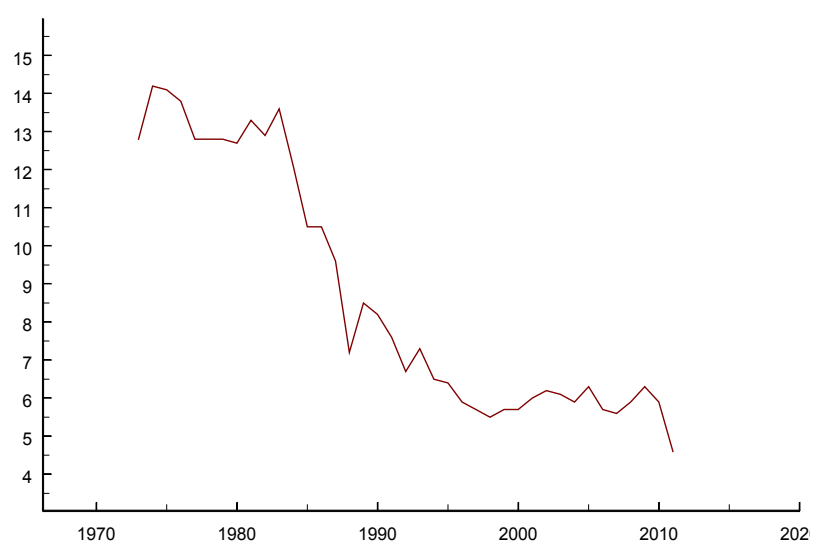

Figure 1: Crude rates per 1000 births of infants with a diagnosis of DDH during the observation period, 1973-2011. 


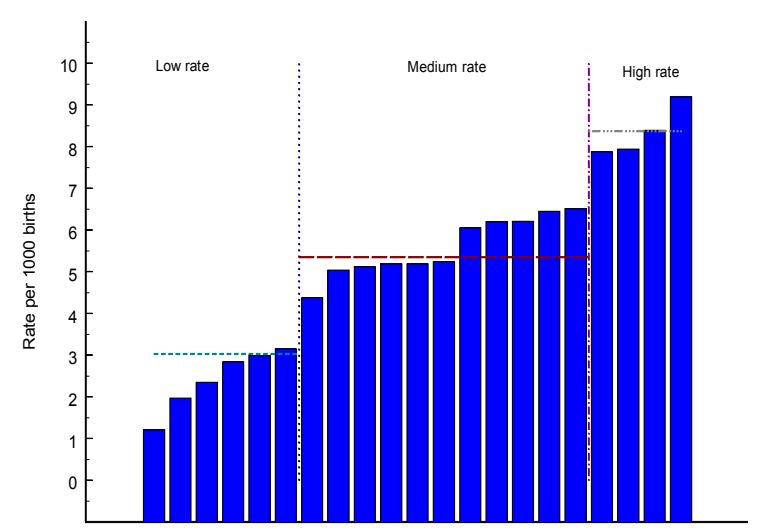

Figure 2: Crude rates of infants with a diagnosis of DDH in each of the Swedish counties, 1998-2011. Divided into three crude groups, used for adjustments.

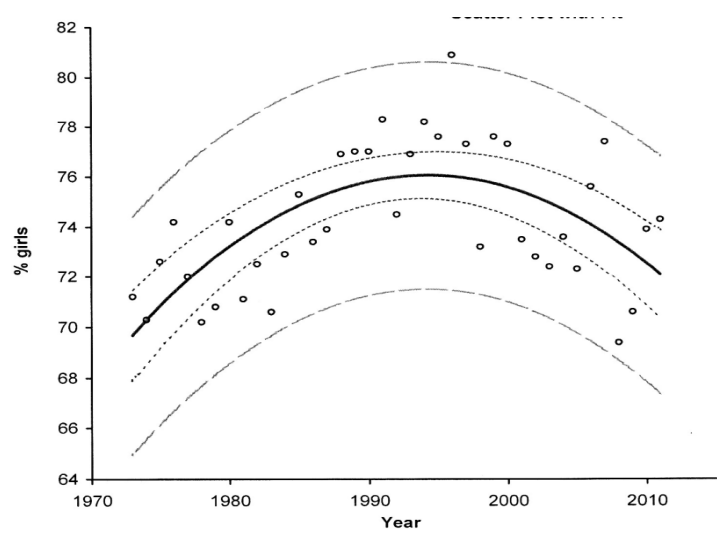

Figure 3: Crude rates of infants with a diagnosis of DDH in each of the Swedish counties, 1998-2011. Divided into three crude groups, used for adjustments.

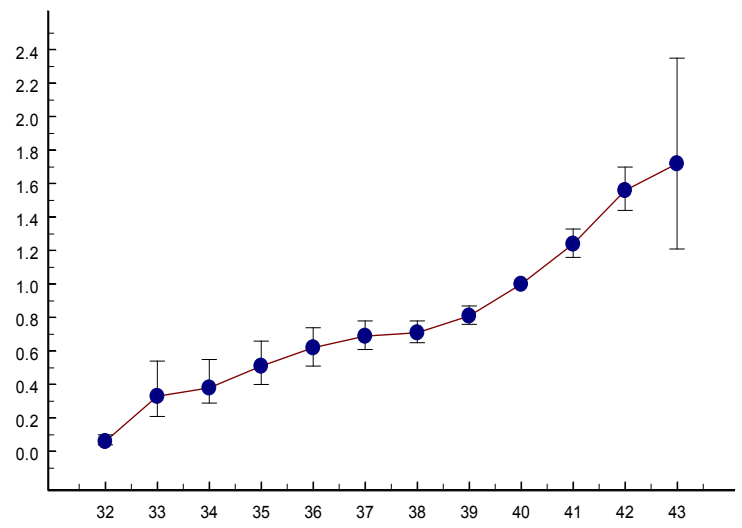

Figure 4: Odds ratio for $\mathrm{DDH}$ at different gestational duration. Vertical lines mark $95 \% \mathrm{Cl}, 1998-2011$.

on odds ratios, adjusted for geography, year of birth, maternal age, parity, smoking, infant sex and breach versus head presentation and are restricted to infants with known smoking and presentation.

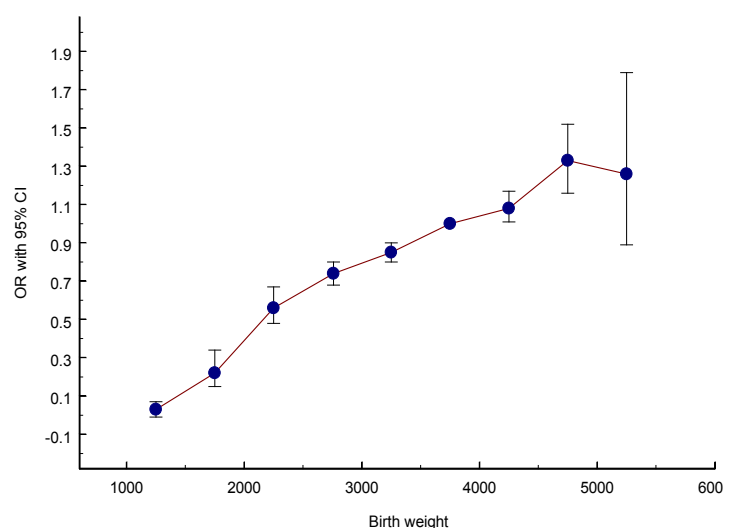

Figure 5: Odds ratio for DDH at different birth weight. Vertical lines mark $95 \%$ Cl, 1998-2011.

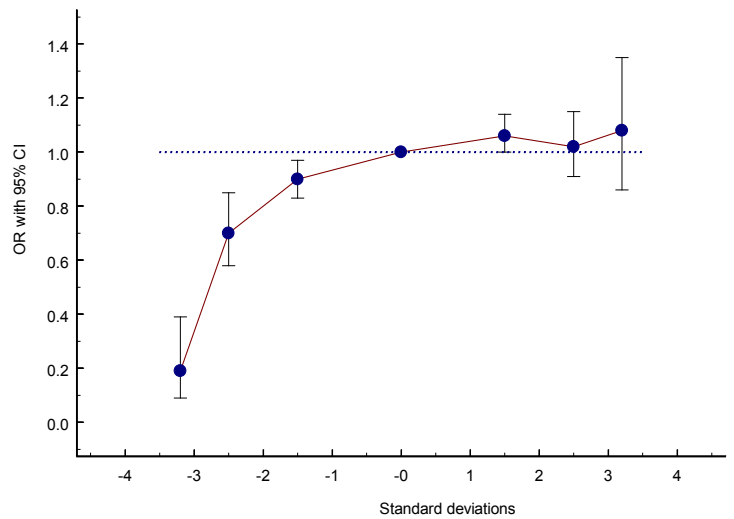

Figure 6: Odds ratio for DDH at different intrauterine growth. Vertical lines mark $95 \% \mathrm{Cl}, 1998-2011$

\begin{tabular}{|l|c|c|c|c|}
\hline & With DDH & Total births & OR & $\mathbf{9 5} \mathbf{~ C l}$ \\
\hline Year of birth & & & & \\
\hline $1998-1999$ & 948 & 169021 & 0.97 & $0.88-1.05$ \\
\hline $2000-2001$ & 1049 & 177002 & 1.00 & $0.92-1.09$ \\
\hline $2002-2003$ & 1164 & 187926 & 1.03 & $0.94-1.11$ \\
\hline $2004-2005$ & 1205 & 197084 & 1.00 & Reference \\
\hline $2006-2007$ & 1176 & 206845 & 0.91 & $0.84-0.99$ \\
\hline $2008-2009$ & 1297 & 213058 & 0.99 & $0.91-1.07$ \\
\hline Maternal age & 1171 & 220570 & 0.86 & $0.87-0-95$ \\
\hline$<20$ & 92 & & & \\
\hline $20-24$ & 832 & 179423 & 0.74 & $0.68-0.80$ \\
\hline $25-29$ & 2474 & 420007 & 1.00 & Reference \\
\hline $30-34$ & 2994 & 475460 & 1.17 & $1.11-1.24$. \\
\hline $35-39$ & 1380 & 227445 & 1.24 & $1.04-1.39$ \\
\hline $40-44$ & 232 & 43070 & 1.20 & $1.24-1.39$ \\
\hline$\geq 45$ & 11 & 1899 & 1.34 & $0.93-2.46$ \\
\hline Parity & & & & \\
\hline 1 & 4312 & 612522 & 1.00 & Reference \\
\hline 2 & 2558 & 495188 & 0.67 & $0.64-0.71$ \\
\hline 3 & 806 & 183668 & 0.55 & $0.51-0.59$ \\
\hline$\geq 4$ & 284 & 80128 & 0.45 & $0.40-0.51$ \\
\hline$E a c 5$ & & & & \\
\hline
\end{tabular}

Each variable adjusted for geography and the other two variables, smoking, and BMI.

Table 2: Year of birth, maternal age and parity as risk factors for $\mathrm{DDH}$. 


\begin{tabular}{|c|c|c|c|c|}
\hline & With dysplasia & Total births & OR & $95 \% \mathrm{Cl}$ \\
\hline \multicolumn{5}{|l|}{ Smoking } \\
\hline Unknown & 530 & 86567 & - & - \\
\hline None & 6966 & 1170499 & 1.00 & Reference \\
\hline$<10$ cigs/day & 381 & 83412 & 0.83 & $0.75-0.92$ \\
\hline$\geq 10$ cigs/day & 113 & 31028 & 0.70 & $0.58-0.85$ \\
\hline \multicolumn{5}{|l|}{ BMI } \\
\hline Unknown & 954 & 164780 & - & - \\
\hline$<18.5$ & 167 & 29244 & 1.00 & $0.85-1.17$ \\
\hline $18.5-24.9$ & 4473 & 740646 & 1.00 & Reference \\
\hline $25-29.9$ & 1716 & 301013 & 0.99 & 0.94-1.05 \\
\hline $30-34.9$ & 502 & 96476 & 0.95 & $0.86-1.04$ \\
\hline$\geq 35$ & 198 & 39247 & 0.93 & $0.81-1.07$ \\
\hline \multicolumn{5}{|c|}{ Unwanted childlessness } \\
\hline 0 & 7326 & 1318355 & 1.00 & Reference \\
\hline 1 year & 210 & 33983 & 0.98 & $0.86-1.13$ \\
\hline 2 years & 208 & 30474 & 0.97 & $0.84-1.11$ \\
\hline 3 years & 113 & 16108 & 0.95 & $0.79-1.15$ \\
\hline 4 years & 53 & 8736 & 0.82 & $0.62-1.07$ \\
\hline
\end{tabular}

Each variable adjusted for geography, year of birth, maternal age, parity and smoking or BMI or smoking or unwanted childlessness.

Table 3: Maternal smoking, body mass index (BMI) and unwanted childlessness as risk factors for $\mathrm{DDH}$.

\begin{tabular}{|c|c|c|c|}
\hline Variable & Adjusted for & OR & $\mathbf{9 5 \%} \mathbf{~ C l}$ \\
\hline \multirow{3}{*}{ Smoking } & Gestational duration & 0.79 & $0.72-0.88$ \\
\cline { 2 - 4 } & Birth weight & 0.81 & $0.73-0.89$ \\
\cline { 2 - 4 } & Intrauterine growth & 0.79 & $0.71-0.87$ \\
\hline \multirow{3}{*}{ Presentation } & Gestational duration & 10.0 & $9.52-10.6$ \\
\cline { 2 - 4 } & Birth weight & 9.01 & $8.55-9.50$ \\
\cline { 2 - 4 } & Intrauterine growth & 8.00 & $7.09-8.47$ \\
\hline
\end{tabular}

*All ORs also adjusted for geography, year of birth, maternal age, infant sex. OR for smoking is also adjusted for presentation, OR for presentation adjusted also for smoking.

Table 4: Breech presentation and maternal smoking as risk factors.

\begin{tabular}{|l|c|c|c|c|}
\hline Drug group & No. with DDH & Total exposed number & OR/RR & $\mathbf{9 5 \%}$ Cl \\
\hline Insulin & 12 & 4163 & $\mathbf{0 . 4 3}$ & $\mathbf{0 . 2 5}-\mathbf{0 . 7 6}$ \\
\hline Gestagens & 33 & 6244 & $\mathbf{0 . 7 1}$ & $\mathbf{0 . 5 0 - 1 . 0 0}$ \\
\hline Anticonvulsants & 32 & 3732 & $\mathbf{1 . 4 8}$ & $\mathbf{1 . 0 4 - 2 . 1 0}$ \\
\hline Valproic acid & 8 & 718 & 2.06 & $0.89-4.05^{\#}$ \\
\hline Carbamazepine & 12 & 1349 & 1.55 & $0.81-2.71^{\#}$ \\
\hline Lamotrigine & 7 & 1212 & 0.92 & $0.37-1.90^{\#}$ \\
\hline Antidepressants & 68 & 21285 & $\mathbf{0 . 5 7}$ & $\mathbf{0 . 4 5 - 0 . 7 2}$ \\
\hline Tricyclic & 9 & 1678 & 0.82 & $0.36-1.52^{\#}$ \\
\hline SSRI & 50 & 17458 & $\mathbf{0 . 5 1}$ & $\mathbf{0 . 3 6}-\mathbf{0 . 6 7}$ \\
\hline SNRI/NRI & 9 & 2542 & 0.62 & $0.32-1.18^{\#}$ \\
\hline Antihistamines & 390 & 74654 & $\mathbf{0 . 8 9}$ & $\mathbf{0 . 8 0 - 0 . 9 9}$ \\
\hline For NVP & 289 & 55048 & 0.90 & $0.80-1.02$ \\
\hline For allergy & 102 & 19731 & 0.86 & $0.71-1.05$ \\
\hline
\end{tabular}

\#RR as observed/expected ratios with exact Poisson 95\% Cl.

Odds ratios (OR) or risk ratios (RR) with $95 \%$ confidence intervals $(95 \% \mathrm{Cl})$. Bold figures mark statistical significance.

Table 5: Effect of maternal use of drugs in early pregnancy and the risk for DDH in the infant. Only drug groups with statistically significant effect.

As maternal smoking and breach presentation may co-vary with gestational duration, the odds ratios for each were calculated, adjusted also for gestational duration (weeks), birth weight (500 g) or intrauterine growth. Table 4 shows the results. The effect of smoking is hardly influenced but the effect of presentation is affected, more by adjustment for intrauterine growth than for gestational duration with birth weight intermediate.

Among infants born 1998-2011 with DDH 18.4 were born in breech presentation while in other infants $3.8 \%$ were (crude $\mathrm{OR}=5.70$, 95\% CI 5.41-6.02). Among infants born in vertex presentation and with $\mathrm{DDH}, 11.1 \%$ were delivered by caesarean section while among other infants $11.5 \%$ were delivered that way. Adjusted OR=1.05 (95\% CI 0.96-1.16). Among infants with breech presentation and DDH $84.5 \%$ were delivered with caesarean section while among other women $88.6 \%$ were delivered that way, adjusted $\mathrm{OR}=0.67$ (95\% CI 0.57-0.78). Adjustment was made for geography, age, parity, infant sex, smoking and pregnancy duration.

\section{Maternal use of drugs and infant DDH}

Table 5 shows the effect of maternal use of some drugs during pregnancy.

Maternal use of insulin was associated with a decreased risk for $\mathrm{DDH}$ in the infant. This effect was reduced but remained significant when the analysis was restricted to term infants $(\mathrm{OR}=0.59,95 \% \mathrm{CI}$ 0.28-0.92). Use of gestagens was also associated with a risk reduction which is marginally significant. When the analysis was restricted to term infants the $\mathrm{OR}$ increased and lost statistical significance $(\mathrm{OR}=0.77$, 95\% CI 0.54-1.10).

The only drug group which is associated with an increased DDH risk was anticonvulsants. This effect was slightly increased when analysis was restricted to term infants ( $\mathrm{OR}=1.56,95 \%$ CI 1.10-2.22). Among the three most commonly used drugs, valproic acids shows the highest and lamotrigine the lowest risk estimate with carbamazepine intermediate but the confidence intervals are wide and the differences between the drugs may be random. It can be noted that among the $32 \mathrm{DDH}$ cases after anticonvulsant exposure, only two had other malformations registered: one had hypospadias and the other a ventricular septum defect.

Use of antidepressants was associated with a reduced risk for $\mathrm{DDH}$, slightly weaker for tricyclic drugs than for SSRI or SNRI/NRI but the difference may be random. The only antidepressant category with a significant reduction of risk was SSRI. The OR did not change when analysis was restricted to term infants.

Antihistamines were also associated with a marginally reduced risk, similar for such drugs used for nausea and vomiting in pregnancy and drugs used for allergy. The effect is similar when the analysis is restricted to term infants ( $\mathrm{OR}=0.91,95 \% \mathrm{CI} 0.81-0.99)$.

\section{Effect of genetics}

Among all women who had infants with DDH during the period studied, 3,045 were themselves born during 1973-1994 and were registered in the Medical Birth Register, 200 had an infant with DDH. Among all female infants born these years $(n=1,099,362)), 18,129$ had $\mathrm{DDH}$. The OR for having an infant with $\mathrm{DDH}$ among the former group of women was 3.69 (95\% CI 3.22-4.22). Adjustment was made for year of birth, maternal age and parity.

\section{Discussion}

This is a large study of epidemiological characteristics of infants with DDH and their mothers. The largest previous study was based on the Norwegian birth register and therefore similar to the present study [6]. The prevalence found in the Norwegian study was 9 per 1000 which 
is slightly lower than the present data for the period 1973-1988. During this period the frequency declined from about 14 to 9 per 1000 in the present material. The Norwegian study comprised 9,955 cases of DDH, the present study 34,547 cases, but the present risk analyses were made on 8,195 cases born after 1997 .

The frequency of the diagnosis of $\mathrm{DDH}$ at birth varies much in different studies and depends on the screening methods used, but true population differences seem to exist [6]. In Sweden physical examination (Ortolani method) dominated up to the 1980s and then gradually this was supplemented with sonographic investigations. As seen in Figure 1 this resulted in a marked reduction in the frequency of diagnosed DDH. From 1998 and onwards, only little annual variation existed, why most risk analyses were performed on infants born 1998-2011.

A major characteristic of infants born with DDH is the strong female excess. If the reduction in frequency had mirrored an exclusion of falsely diagnosed cases, one would have expected an increase in the female excess with decreasing frequency. This was indicated up to about 1995 but then the female excess declined while the frequency remained mainly unchanged. The change in the percentage of females was, however, moderate but statistically significant: from a low $70 \%$ to a maximum of about $75 \%$ and back to $72 \%$. The explanation to this variation is unclear but could mirror changes in the application of diagnostic tools.

A marked variation in frequency could also be seen between different counties and this did not correspond to changes in female frequency. It is unknown if these differences in frequency mirror differences in diagnosis, perhaps explainable by different policies of sonographic diagnosis, or if it is only a matter of registration differences. There was a large variation in rates also between different hospitals and a general weak trend that the rate increased with the size of the hospital. One explanation to this trend could be a referral for delivery of pregnancies with breech presentation from small to larger hospitals.

The actual frequency of DDH is thus variable but this will not much affect risk estimates. As differences may exist between different counties in, for instance, the distribution of maternal characteristics, adjustment for geography has been made, using low, medium and high frequency as explanatory variable.

Some risk factors are well known from the literature and have also been demonstrated in the present investigation: female excess, breech presentation, first born and family history (here studied as DDH in mothers when newborn and in siblings) [1-6]. In the present study female sex was a strong risk factor (2.7 times more often a girl than a boy) comparable with the 2.54 risk ratio found in a meta-analysis [1]. The crude OR for breach presentation (5.70) was somewhat higher than that obtained from the meta-analysis, 3.75 [1]. As shown in the present study, the use of caesarean sections at breech presentation will affect the risk in this situation The relative risk for being first born according to the meta-analysis was 1.44 , similar to the risk for parity 1 compared with parity 2 in the present material (1.50) but in this material a further risk reduction was seen for parities 3 and 4+. A positive family history gave a relative risk of 1.30 in the meta-analysis while the specific association between maternal DDH and infant DDH was much stronger in the present investigation: 3.69 and the sibling risk were also much higher. The last risk factor studied by the meta-analysis was left side-our data did not permit analysis of side of anomaly at unilateral conditions.
Most infants with DDH had no other congenital malformation detected in the newborn, but among those who had (4.5\% against 3\% in the total population); an over-representation was seen of varying degree for specific types. The strongest association was seen with foot deformities which have been repeatedly pointed out in the literature [7]. A high risk estimate is also seen for CNS malformations which are likely due to neurological disturbances.

The relationship between gestational duration or birth weight and the risk for DDH was clear-cut and confirms those studies in the literature which found a reduced risk at preterm birth. Also intrauterine growth (as measured as standard deviations from expected birth weight for gestational age) was of importance but an effect was mainly seen at growth retardation.

A clear-cut although not very strong association between maternal age and an increased risk for DDH was found. Maternal age is usually not mentioned as a risk factor but the impact of low parity confounds the analyses. In the present study adjustment for parity was made.

The present analysis identified maternal smoking as a "protective" factor for DDH. This finding agrees with the results of a clinical and sonographic study made on the relationship between maternal smoking and a decreased risk of signs or DDH [8]. That paper found effects only on female infants but in the present study the effect of maternal smoking was similar for the two sexes. As maternal smoking can influence other risk factors like pregnancy duration and intrauterine growth, adjustment for such factors were made in the present study but they hardly affected the OR estimates. It seems likely that smoking has another and more direct effect, perhaps by changing the endocrine environment of the fetus [8]. The beneficial effect on DDH does not, however, justify the many other harmful effects of maternal smoking on the newborn.

Caesarean section has no noticeable effect on the DDH risk at vertex presentation but reduces markedly the risk at breech presentation. This shows that the actual vaginal birth process of an infant in breech presentation but not of an infant in vertex presentation contributes to the cause of DDH. The finding contrast to some previous results in the literature [6].

There is only one drug group which could be identified as increasing the risk for DDH, namely, anticonvulsants. Such drugs often have a general teratogenicity and may also affect acetabular development. The majority of the anticonvulsant exposed infants with DDH had no other congenital malformation (the two exceptions had hypospadias or ventricular septum defects) so the association is hardly a secondary effect of other teratogenic phenomena caused by these drugs.

On the other hand, a reduction of the DDH risk was seen for some other drug categories. Some of them will decrease gestational duration or intrauterine growth and in this way can reduce the risk but little change of the ORs was seen when analysis was restricted to term infants. Such "protective" effects were seen for insulin, gestagens, antidepressants, and antihistamines but the statistical significances for gestagens and antihistamines were marginal. The mechanism behind these effects is unknown.

In summary, the analysis has pointed out some previously less well known risk factors for DDH: maternal age (when adjusted for parity) and maternal use of anticonvulsants. Maternal smoking reduces the risk in both sexes and so does the use of insulin or antidepressants, 
Citation: Kallen B (2014) Risk Factors for Developmental Dysplasia of the Hip in the Newborn Data from the Swedish Medical Birth Register. Clinics Mother Child Health 11: 153. doi: 10.4172/2090-7214.1000153

Page 6 of 6

perhaps also use of gestagens or antihistamines. Caesarean section does not affect the risk at vertex presentation but reduces it considerably at breech presentation.

\section{Acknowledgement}

My thanks to the National Board of Health and Welfare, Stockholm, Sweden, for giving I access to the data files. The study was supported by a grant from Evy and Gunnar Sandberg Foundation, Lund, Sweden.

\section{References}

1. Ortiz-Neira CL, Oddone Paolucci E, Donnon T (2012) A meta-analysis of common risk factors associated with the diagnosis of developmental dysplasia of the hip in newborns. Eur J Radiol 81: 344-351.

2. De Hundt M, Vlemmix F, Bais JMJ, de Groot CJ, et al. (2012) Risk factors for developmental dysplasia of the hip: a meta-analysis. Eur J Obstet Gynecol Reprod Biol 165: 8-17.
3. Ishikawa N (2008) The relationship between neonatal developmental dysplasia of the hip and maternal hyperthyroidism. J Ped Orthop 28. 432-434.

4. National Board of Health and Welfare. Centre for Epidemiology. The Swedish Medical Birth Register - a summary of content and quality.

5. Kallen B (1995) Birth weight for gestational age standard based on data in the Swedish Medical Birth Register, 1985-1989. Eur J Epidemiol 11: 601-606

6. Hinderaker T, Daltveit AK, Irgens LM, Liden A, Reikeras O (1994) The impact of intra-uterine factors on neonatal hip instability. Acta Orthop Scand 65: 239-242.

7. Bracken J, Tran T, Ditchfield M (2012) Developmental dysplasia of the hip Controversies and current concepts. J Pediatr Child Healh 48: 963-973.

8. Chasiotis-Tourikis E, Varvarigou A, Yarmenitis S, Vandoros N, Beratis NG (2003) Maternal smoking during pregnancy improves the anatomy of the hip joint in the female neonate. J Matern Fetal Neonatal Med 14: 45-50. 